\title{
P1.7 Troubles sensoriels et désafférentation
}

\author{
(C) Springer-Verlag France 2011
}

\section{P1-7.36}

Olfaction dans la maladie de Parkinson du sujet âgé : étude des interactions olfactives et trigéminales - résultats préliminaires

C. Foguem ${ }^{1,2}$, A. Doe De Mandreville ${ }^{3}$, T. Moulin ${ }^{2,4}$, P. Vandel ${ }^{2,5}$, G. Brand ${ }^{2}$, M. Dramé, P. Pfitzenmeyer, F. Blanchard

${ }^{1}$ Service de Médecine Interne et Gérontologie clinique, Hôpital Maison Blanche, $\mathrm{CHU}$ de Reims, Reims

${ }^{2}$ Laboratoire de Neurosciences (Ea 481), Université de Franche Comté, Besançon

${ }^{3}$ Service de Neurologie, Hôpital Maison Blanche, CHU de Reims, Reims ${ }^{4}$ Service de Neurologie, Hôpital Jean-Minjoz, CHU de Besançon

${ }^{5}$ Service de Psychiatrie, Hôpital Saint-Jacques, CHU de Besançon, Besançon, France

Introduction: La maladie de Parkinson idiopathique (MPI), est la deuxiéme affection dégénérative la plus fréquente après la maladie d'Alzheimer; elle affecte 2 à $3 \%$ des personnes âgées de plus de 75 ans. La MPI affecte le contrôle des nerfs moteurs engendrant les signes cliniques cardinaux de la maladie : tremblement de repos, bradykinésie, rigidité auxquels s'ajoutent l'asymétrie, l'absence de signe atypique, l'absence d'autre étiologie et une réponse marquée à la L-Dopa. La MPI affecte également la mémoire et le système sensitif. Parmi les atteintes sensitives associées à la MPI l'atteinte olfactive serait la plus étudiée. Objectifs: Dans la mesure où la plupart des substances odorantes activent à la fois les systèmes olfactif $(\mathrm{CN} \mathrm{I})$ et trigéminal $(\mathrm{CN} \mathrm{V})$, il nous est apparu opportun de déterminer la part respective de chacun (CN I et $\mathrm{CN}$ V) dans la chémoréception nasale. L'objectif du présent essai était de caractériser les dysfonctions olfactive et trigéminale ( $\mathrm{CN}$ I et $\mathrm{CN} \mathrm{V}$ ) dans la chémoréception nasale chez des patients âgés de plus de 65 ans atteints de MPI. L'hypothèse principale étant que la sensibilité trigéminale serait peu déficitaire et ne permettrait pas de différencier une population âgée normale d'une population de patients atteints de la MPI. Méthodes: Au cours de cette étude prospective, des patients âgés de plus de 65 ans atteints de MPI, n'ayant pas de tabagisme actif, d' AVC récent ni d'infection de la sphère ORL ou broncho-pulmonaire, hospitalisés ou vus en consultation de mars à fin mai 2011 au CHU de Reims, ont été inclus dans l'étude. Ils ont été comparés avec des sujets contrôles sains, appareillés sur des caractéristiques épidémiologiques pour la détection des stimuli olfactifs dilués à partir de 2 odeurs. L étude a utilisée des stimuli dans une gamme allant de molécules n'activant que le nerf I, l'alcool phényl éthylique (PEA), de molécules activant conjointement les nerfs I et $\mathrm{V}$, le Butanol (BUT). Résultats: 22 sujets ont été inclus dont 11 patients souffrant de la MPI : 6 hommes et 5 femmes ; ainsi que 6 hommes et 5 femmes de plus de 65 ans en bonne santé répondant aux critères d'inclusion. Ils en ressort que les témoins à âges égales ont un meilleur seuil de détection des stimuli que les patients atteints de MPI; que parmi les patients atteints de MPI le seuil de sensibilité du PEA est moins bon qu'avec le BUT, comparés aux témoins. Les femmes ont également une meilleure olfaction que les hommes.
Conclusion: Certaines récentes études neuropathologiques mettent l'accent sur l'atteinte olfactive précoce et sévère dans la MPI, dans lesquelles il est mis en évidence un déficit dopaminergique marqué au niveau du bulbe olfactif. D'autres études cliniques ont rapporté que l'atteinte olfactive dans la MPI est bilatérale, indépendamment du stade ou de la classification de la maladie et du traitement antiparkinsonien. D'après nos résultats préliminaires le déclin de la sensibilité trigéminale, serait nettement moins marqué que celui de la sensibilité olfactive et probablement moins tributaire de la MPI.

\section{P1-7.37}

Presbyproprioception? Les effets du vieillissement sur les aspects comportementaux et attentionnels de la proprioception

M. P. Boisgontier ${ }^{1}$, I. Olivier ${ }^{1}$, V. Nougier ${ }^{1}$

${ }^{I}$ Neuroscience, UJF-Grenoble 1 / CNRS / TIMC-IMAG UMR 5525, La Tronche Cedex, France

Introduction: Les effets du vieillissement physiologique sur la proprioception ont largement été testés dans la littérature. Cependant, la divergence des résultats obtenus ne permet pas de conclure sur la présence ou l'absence de ces effets. Objectifs: Avec pour objectif d'expliquer ces divergences, cette étude se proposait d'évaluer les effets du vieillissement non seulement sur les aspects comportementaux de la proprioception mais également sur le coût attentionnel lié à ces comportements. Méthodes: Pour ce faire, 15 sujets âgés et 15 sujets jeunes se sont portés volontaires pour participer à cette étude. Ces sujets ont réalisé une tâche de mise en correspondance des positions articulaires des chevilles dans des paradigmes de simple tâche et de double tâche. La tâche de mise en correspondance était la tâche dite principale et devait être réalisée de la même manière quelque soit la condition. La tâche cognitive dite secondaire devait être réalisée du mieux possible sans affecter la réalisation de la tâche primaire. Les erreurs totale, variable et constante étaient calculées pour la tâche proprioceptive et utilisées pour évaluer la performance comportementale des sujets. La mesure dans laquelle la performance à la tâche cognitive secondaire diminuait dans le protocole de double tâche était utilisée pour rendre compte du coût attentionnel requis pour réaliser la tâche proprioceptive. Résultats: Les résultats ne montrent pas de différence de performance proprioceptive en termes de comportement entre les sujets jeunes et âgés dans un paradigme de simple tâche. Cependant, la réalisation d'une tâche cognitive concurrente de la tâche proprioceptive révèle un coût attentionnel plus important de la tâche proprioceptive pour les personnes âgées par rapport aux sujets jeunes. Conclusion: Ces résultats suggèrent que la proprioception est altérée du fait du vieillissement physiologique, sinon en termes de comportement, au moins en termes de coût attentionnel. 